\title{
The influence of fission on neutron star merger r-process and the position of the third r-process peak
}

\author{
M. Eichler ${ }^{* a}$, A. Arcones ${ }^{b c}$, A. Kelic ${ }^{c}$, O. Korobkin ${ }^{d}$, K. Langanke ${ }^{b c}$, G. \\ Martinez-Pinedo $^{b c}$, I. V. Panov ${ }^{e a}$, T. Rauscher ${ }^{f a}$, S. Rosswog ${ }^{d}$, C. Winteler ${ }^{g}$, N. T. \\ Zinner $^{h}$, and F.-K. Thielemann ${ }^{a}$ \\ ${ }^{a}$ Department of Physics, University of Basel, Switzerland \\ ${ }^{b}$ Institut für Kernphysik, Technische Universität Darmstadt, Germany \\ ${ }^{c}$ GSI Helmholtzzentrum für Schwerionenforschung GmbH, Darmstadt, Germany \\ ${ }^{d}$ The Oskar Klein Centre, Department of Astronomy, Stockholm University, Sweden \\ ${ }^{e}$ SSC RF ITEP of NRC "Kurchatov Institute", Moscow, Russia \\ ${ }^{f}$ Centre for Astrophysics Research, University of Hertfordshire, UK \\ ${ }^{g}$ Institut Energie am Bau, Fachhochschule Nordwestschweiz, Muttenz, Switzerland \\ ${ }^{h}$ Department of Physics and Astronomy, Aarhus University, Denmark \\ E-mail: marius.eichler@unibas.ch
}

The comparison between observational abundance features and those obtained from nucleosynthesis predictions of stellar evolution and/or explosion simulations can scrutinize two aspects: (a) the conditions in the astrophysical production site and (b) the quality of the nuclear physics input utilized. Here we test the abundance features of r-process nucleosynthesis calculations using four different fission fragment distribution models. Furthermore, we explore the origin of a shift in the third r-process peak position in comparison with the solar r-process abundances which has been noticed in a number of merger nucleosynthesis predictions. We show that this shift occurs during the r-process freeze-out when neutron captures and $\beta$-decays compete and an $(\mathrm{n}, \gamma)-(\gamma, \mathrm{n})$ equilibrium is not maintained anymore. During this phase neutrons originate mainly from fission of material above $A=240$. We also demonstrate that a faster (and thus earlier) release of these neutrons, e.g., by shorter $\beta$-decay half-lives of nuclei with $\mathrm{Z}>80$, as suggested by recent theoretical advances, can partially prevent this shift.

XIII Nuclei in the Cosmos,

7-11 July, 2014

Debrecen, Hungary

\footnotetext{
*Speaker.
} 


\section{Introduction}

The astrophysical production site(s) of the rapid neutron capture process (r-process) are still unkown. Observations of metal-poor stars reveal that there may be more than one site and that the r-process elements can be divided into two categories: a "weak" component responsible for the production of the lighter elements, and a "strong" component, which produces the heavier elements and shows a remarkable robustness in the abundance pattern [1]. Neutron star mergers (NSM) are a viable candidate for the production of the strong r-process component [2, 3, 4]. In such an extremely neutron-rich environment, the neutron-to-seed ratio can reach 1000 and the reaction path includes several fission cycles, which leads to a robust final abundance distribution. Therefore, the fission treatment becomes an important part of nucleosynthesis calculations in NSMs. As there are not many experimental data that are relevant for fissioning nuclei on the r-process path, there exist many different predictions for the fission barriers and the fragment distributions. We explore different models and show that the choice of (a set of) fission barriers and fission fragment distribution model has a large impact on the final abundance distribution.

\subsection{Nuclear mass models and fission fragment distribution models}

Due to its nature the r-process operates in the extremely neutron-rich part of the nuclear chart. The masses of most nuclei on the reaction path are not (yet) determined experimentally and one has to rely on predictions based on a nuclear mass model. Mass models can differ substantially in their extrapolations towards the neutron drip-line and in their fission barrier predictions [5]. Therefore, the use of different mass models gives rise to different abundance distributions, underlining the large nuclear uncertainties that are still present in r-process nucleosynthesis calculations. Fission fragment distribution models are used to statistically predict the fission fragment yields of each fissioning nucleus. While older models often use simple parametrizations, more sophisticated models are tested on known fission data and take into account shell effects of parent nuclei and fragments. In each fission reaction, there is a possibility of fission neutrons to be emitted. For experimentally
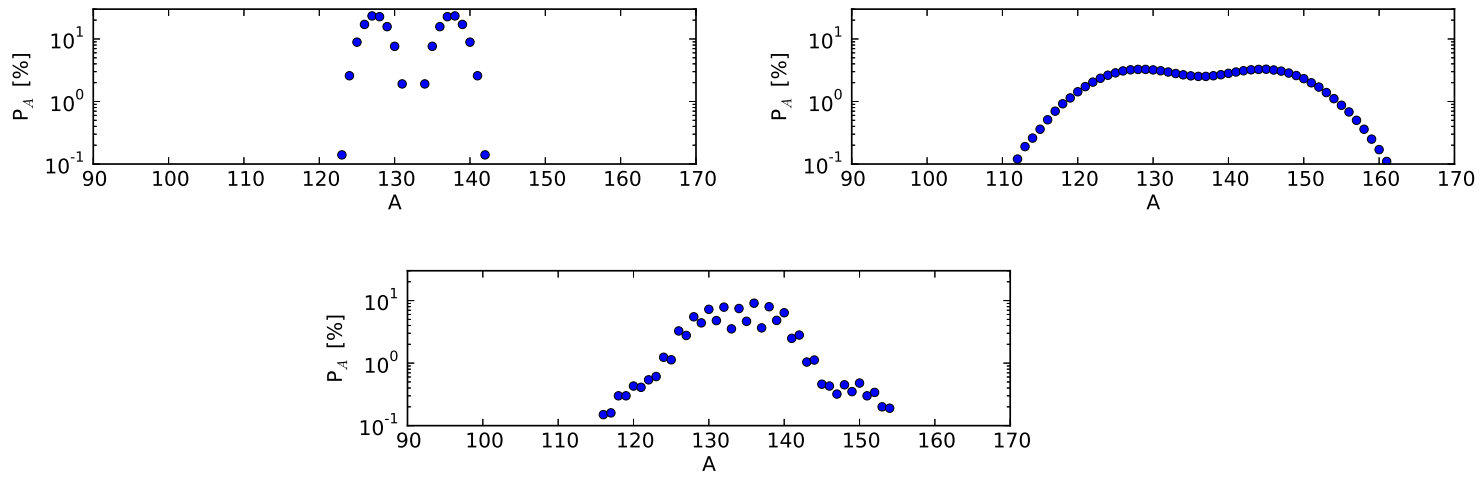

Figure 1: Fission fragment distributions for the models considered in our calculations, here for the case of neutron-induced fission of ${ }^{274} \mathrm{Pu}$ (top left: Panov et al. 2008 [9], top right: Kodama \& Takahashi 1975 [7], bottom: ABLA07 (Kelic et al. 2008) [10]). For this reaction Panov et al. (2008) predicts 9, ABLA07 7 released fission neutrons. Kodama \& Takahashi (1975) and Panov et al. (2001) do not predict any fission neutrons. The distribution for the latter model consists only of two products with $A_{1}=130$ and $A_{2}=144$. 
studied fission reactions, the number of fission neutrons has been found to be $2-4$, but it is known to increase with mass number as heavy nuclei become more neutron-rich [6]. If a daughter nucleus is very neutron-rich, additional neutrons can be emitted via photodissociations. In this work we use and compare four different fission fragment distribution models that vary in complexity: (a) Kodama \& Takahashi (1975) [7], (b) Panov et al. (2001) [8], (c) Panov et al. (2008) [9], and (d) ABLA07 (Kelic et al. 2008) [10]. The fragment yields predicted by these models on the example of fissioning ${ }^{274} \mathrm{Pu}$ are shown in Figure 1. It can be seen that not only the range of possible fragments differs considerably in each model, but also the predicted amount of fission neutrons.

\section{Method}

Our nucleosynthesis calculations are based on a NSM simulation with two $1.4 \mathrm{M}_{\odot}$ neutron stars from Rosswog et al. (2013) [11]. We use 30 representative fluid trajectories, covering all the conditions in the ejected matter and providing the temperature, density and electron fraction within the ejected material up to a time of $t_{0}=13 \mathrm{~ms}$. To account for the expansion and cooling phase, we extrapolate using the following prescriptions:

$$
\begin{aligned}
& \rho(t)=\rho_{0}\left(\frac{t}{t_{0}}\right)^{-3} \\
& T(t)=T\left[S, \rho(t), Y_{e}(t)\right],
\end{aligned}
$$

with time $t$, density $\rho$, temperature $T$, entropy $S$ and $Y_{e}$ being the electron fraction of the fluid element. The index 0 denotes the values at $t_{0}$. The temperature is calculated at each timestep using the equation of state of Timmes \& Swesty (2000) [12].

The calculations are performed using the nuclear network WINNET [13], including over 6000 isotopes between stability and neutron drip-line. The set of reaction rates utilized is based on masses from the Finite Range Droplet Model (FRDM) [14], and the Extended Thomas Fermi Model with Strutinsky Integral (with shell quenching) (ETFSI-Q) [15, 16], in combination with the statistical model calculations of Rauscher \& Thielemann (2000) [17] for $Z \leq 83$, and the Hartree-FockBogoliubov Model (HFB-14) [18], respectively. Theoretical $\beta$-decay rates are taken from Moeller et al. (2003) [19], experimental data from the nuclear database NuDat2 [20]. The neutron capture rates on heavy nuclei $(Z>83)$ as well as the neutron-induced fission rates are from Panov et al. (2010) [21], while the $\beta$-delayed fission rates are taken from Panov et al. (2005) [22].

\section{Results}

Final abundance distributions for each fission fragment distributions model are shown in Figure 2 for the FRDM mass model. The differences in the models are clearly reflected in the shape of the second r-process peak (mass range $120<\mathrm{A}<140$ ) in the final abundances: The two models with the narrowest distribution range (Panov et al. 2001 \& 2008) produce a distinct peak, followed by a region of underproduction compared to the solar r-abundances between $140<\mathrm{A}$ $<170$. The Kodama \& Takahashi model, on the other hand, features an extremely broad distribution of fission fragments, which leads to an overproduction of nuclei beyond the second peak. The ABLA07 model shows the best overall agreement with the solar r-abundances. The remaining 

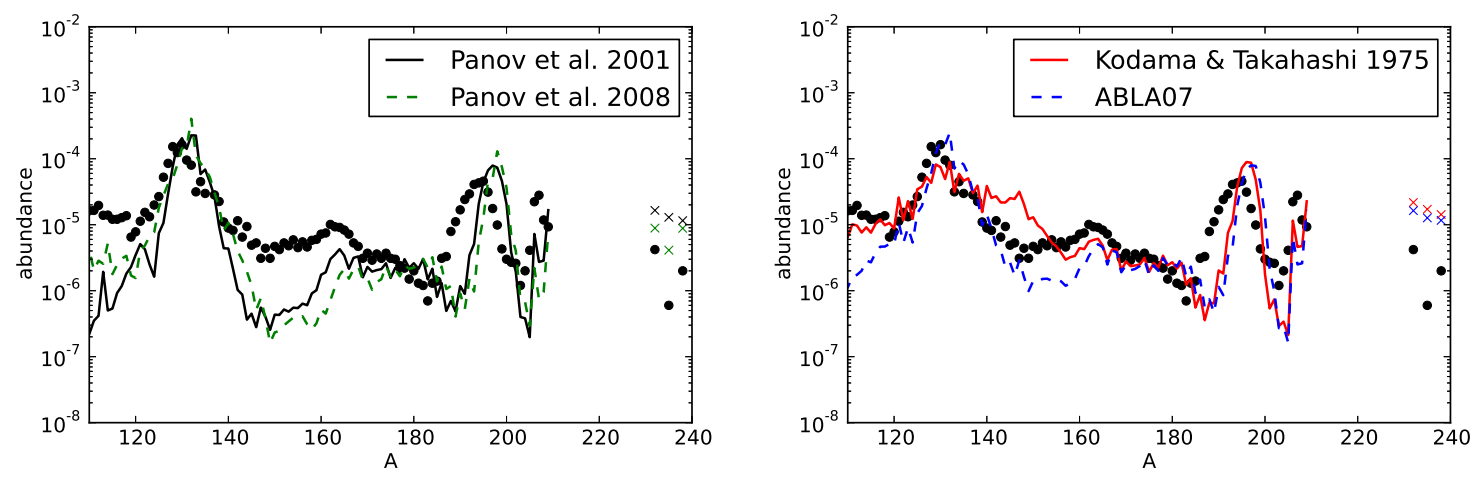

Figure 2: Final abundances of the integrated ejecta around the second and third peak for a NSM [11] at a simulation time $t=10^{6} \mathrm{~s}$, employing the FRDM mass model combined with four different fission fragment distribution models (see text). For reasons of clarity the results are presented in two graphs. The abundances for Th and $\mathrm{U}$ are indicated by crosses. The dots represent the solar r-process abundance pattern [23].

underproduction of $140<\mathrm{A}<170$ nuclei is an effect of the mass model and did not appear when we repeated the calculations with the ETFSI-Q and the HFB-14 models in combination with the ABLA07 model.

\subsection{The position of the third peak}

In the following we would like to discuss one remarkable issue that is present in Figure 2: The position of the third peak in our final abundance distributions is always shifted towards heavier mass numbers compared to the solar distribution. This shift is also present in other recent publications on NSM nucleosynthesis and, as can be seen in Figure 2, the fission fragment distribution model seems to have an impact on the magnitude of the shift. In addition, we observe that the third peak is still in line with the solar abundance peak at the time of r-process freeze-out (defined as the time where the averaged $(n, \gamma)$ and $(\gamma, n)$ timescales becomes larger than the averaged $\beta$-decay timescale), with the shift happening only later. It is caused by late neutron captures by third peak nuclei, the neutrons being continuously supplied by the fissioning of material above $\mathrm{A} \simeq 240$. It should be noted that not only the neutrons directly released from fission are relevant, but also those released by the fission products as they decay to the r-process path. Furthermore, mainly nuclei beyond the second peak are affected, since (a) the neutron capture cross sections depend on the mass number of the capturing nuclei and are generally larger for higher mass numbers, and (b) the abundance pattern of the second peak is dominated by the fission fragment production, even after the r-process freeze-out.

Recent $\beta$-decay calculations predict considerably shorter half-lives for nuclei with $\mathrm{Z}>80$ by factors of 10 or even more $[24,25]$. We have tested the effect of these findings on our nucleosynthesis calculations by accelerating the $\beta$-decay rates of all $\mathrm{Z}>80$ nuclei by (constant) factors of 2.5 and 6, respectively. The results are shown in Figure 3 for the example of one trajectory. As a consequence of the increased $\beta$-decay rates, the reaction flux for the heavy nuclei is accelerated, which increases both the heating rate and the temperature at around $0.1 \mathrm{~s}$ in the calculation (Fig. 3a). Additionally, the release of neutrons by fission of heavy nuclei is accelerated, providing neutrons before freeze-out (when the third r-process peak is still located close to solar values). The 

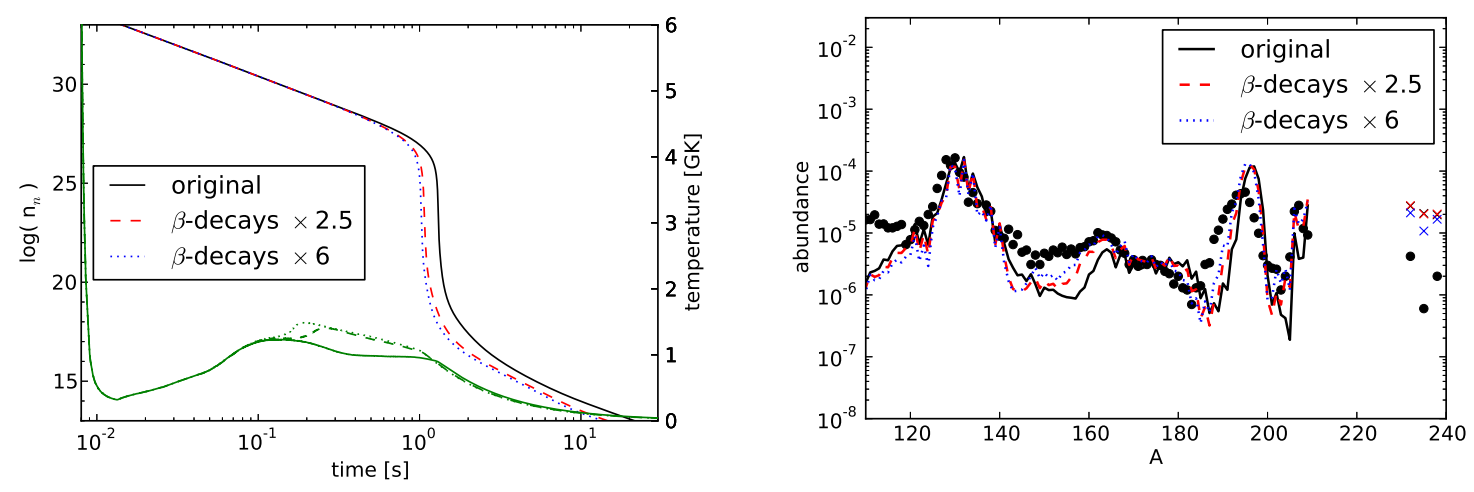

Figure 3: Neutron density $\left(n_{n}\right)$ and temperature (green lines in the bottom part of the graphs; the linestyles correspond to the individual calculations) as well as final abundances for a NSM calculation with accelerated $\beta$-decays compared to the original calculation (solid line) with the FRDM nuclear mass model. Here we use the ABLA07 fission fragment distribution model. See text for further explanations.

evolution after freeze-out proceeds faster and consequently the period of time where a combination of neutron captures and $\beta$-decays can move nuclei to higher mass numbers becomes shorter. As a consequence, the shift in the third r-process peak is reduced. Figure 3 shows that not only the third peak is affected by the modified $\beta$-decay rates, but also the rare earth peak. In order to test our findings, we have repeated our calculations with two different mass models, ETFSI-Q $[15,16]$ and HFB-14 [18]. Although for those cases the abundance distribution differs due to different masses along the r-process path, the shift of the third peak is also apparent and modifying the $\beta$-decay rates of the $Z>80$ nuclei as above leads to the same result. We therefore assume that the position of the third peak is determined mainly by the combination of neutron density and temperature at and beyond the time of the r-process freeze-out and not by the nuclear input.

\section{Conclusions}

We have shown that the r-process yields in neutron star merger (NSM) ejecta are strongly affected by the adopted model for fission fragment distributions. Among the models utilized here, we find that the best agreement with the r-process pattern of solar abundances is achieved with the ABLA07 model, which was tested not only for experimental fission fragment distrubutions, but also for the fragment distributions from extended heavy ion collision yields and thus goes far beyond earlier pure extrapolations of experimental fission fragment data. Similar studies with different fission fragment distribution models have been performed recently [26].

In neutron-rich NSM nucleosynthesis, the third peak in the final abundance distribution shifts towards higher masses if after the r-process freeze-out the conditions for further neutron capture reactions prevail. The two main factors that affect these conditions are temperature and neutron density. If the neutron density is high enough, several neutron captures after freeze-out shift the peak. This can be prevented by photodissociations if the temperature is sufficiently high. Motivated by recent theoretical predictions [24, 25], we have explored the effect of increased $\beta$-decay rates for the heaviest nuclei in our network ( $\mathrm{Z} \geq 80$ ). In this case the reaction flux is accelerated, leading to 
an earlier release of the fission (and $\beta$-delayed) neutrons, where they are recycled in the $(\mathrm{n}, \gamma)-(\gamma, \mathrm{n})$ equilibrium that is present before the freeze-out.

\section{References}

[1] Sneden, C., Lawler, J. E., Cowan, J. J., Ivans, I. I., \& Den Hartog, E. A. 2009, ApJS, 182, 80

[2] Freiburghaus, C., Rosswog, S., \& Thielemann, F.-K. 1999, ApJ, 525, L121

[3] Bauswein, A., Goriely, S., \& Janka, H.-T. 2013, ApJ, 773, 78

[4] Rosswog, S., Korobkin, O., Arcones, A., Thielemann, F.-K., \& Piran, T. 2014, MNRAS, 439, 744

[5] Petermann, I., Langanke, K., Martinez-Pinedo, G., Panov, I. V., Reinhard, P.-G., \& Thielemann, F.-K. 2012, European Physical Journal A, 48, 122

[6] Steinberg, E. P. \& Wilkins, B. D. 1978, ApJ, 223, 1000

[7] Kodama, T. \& Takahashi, K. 1975, NuPhA, 239, 489

[8] Panov, I. V., Freiburghaus, C., \& Thielemann, F.-K. 2001, Nuclear Physics A, 688, 587

[9] Panov, I. V., Korneev, I. Y., \& Thielemann, F.-K. 2008, Astronomy Letters, 34, 189

[10] Kelic, A., Ricciardi, M. V., \& Schmidt, K.-H. 2008, Proceedings: Dynamical Aspects of Nuclear Fission, World Scientific, 6, p. 203

[11] Rosswog, S., Piran, T., \& Nakar, E. 2013, MNRAS, 430, 2585

[12] Timmes, F. X. \& Swesty, F. D. 2000, ApJS, 126, 501

[13] Winteler, C., Käppeli, R., Perego, A., Arcones, A., Vasset, N., Nishimura, N., Liebendörfer, M., \& Thielemann, F.-K. 2012, ApJL, 750, L22

[14] Möller, P., Nix, J. R., Myers, W. D., \& Swiatecki, W. J. 1995, Atomic Data and Nuclear Data Tables, 59,185

[15] Aboussir, Y., Pearson, J. M., Dutta, A. K., \& Tondeur, F. 1995, Atomic Data and Nuclear Data Tables, 61,127

[16] Pearson, J. M., Nayak, R. C., \& Goriely, S. 1996, Physics Letters B, 387, 455

[17] Rauscher, T. \& Thielemann, F.-K. 2000, Atomic Data and Nuclear Data Tables, 75, 1

[18] Goriely, S., Hilaire, S., \& Koning, A. J. 2008, A\&A, 487, 767

[19] Möller, P., Pfeiffer, B., \& Kratz, K.-L. 2003, Phys. Rev. C, 67, 055802

[20] NuDat2. 2009, National Nuclear Data Center, information extracted from the NuDat 2 database, URL http://www.nndc.bnl.gov/nudat2/

[21] Panov, I. V., Korneev, I. Y., Rauscher, T., Martinez-Pinedo, G., Kelic-Heil, A., Zinner, N. T., \& Thielemann, F.-K. 2010, A\&A, 513, A61

[22] Panov, I. V., Kolbe, E., Pfeiffer, B., Rauscher, T., Kratz, K.-L., \& Thielemann, F.-K. 2005, Nuclear Physics A, 747, 633

[23] Sneden, C., Cowan, J. J., \& Gallino, R. 2008, Ann.Rev.Astron.Astrophys., 46, 241

[24] Panov, I. V., Lutostansky, Y. S., \& Thielemann, F. K. 2014, Bull. Russ. Acad. Sci. Phys., in press

[25] Marketin, T., Huther, L., \& Martinez-Pinedo, G. 2014, in preparation

[26] Goriely, S., Sida, J.-L., Lemaitre, J.-F., Panebianco, S., Dubray, N., Hilaire, S., Bauswein, A., \& Janka, H.-T. 2013, Physical Review Letters, 111, 242502 